\title{
USING MACHINE LEARNING TO BUILD A SEMI-INTELLIGENT BOT
}

\author{
Ali Rahmani, Patrick Laffitte, Raja Haddad and Yassin Chabeb \\ Research and development entity, Data Science team, Palo IT, Paris, France
}

\begin{abstract}
Nowadays, real-time systems and intelligent systems offer more and more control interface based on voice recognition or human language recognition. Robots and drones will soon be mainly controlled by voice. Other robots will integrate bots to interact with their users, this can be useful both in industry and entertainment. At first, researchers were digging on the side of "ontology reasoning". Given all the technical constraints brought by the treatment of ontologies, an interesting solution has emerged in last years: the construction of a model based on machine learning to connect a human language to a knowledge base (based for example on RDF). We present in this paper our contribution to build a bot that could be used on real-time systems and drones/robots, using recent machine learning technologies.
\end{abstract}

\section{KEYWORDS}

Real-time systems, Intelligent systems, Machine learning, Bot

\section{INTRODUCTION}

We present here our contributions within Palo IT [17] for a year of research \& development activities. The main part of the R\&D entity works on Data Science trends and intelligent systems based on machine learning technologies. The aim of this project was to create a semi-intelligent bot. This bot must be able to analyse facts, reason and answer questions using machine learning methods. This paper aims to provide an overview on our work during this project. It consists of four parts. In the first part, we present the context of the project, the problematics, some related works, the objectives. In the second part, we present details of the tasks that we deal with during our research project. Tasks about the implementation and testing of different methods of text mining - by applying these methods on text data in French - and the test results are detailed in the third part of this paper. Finally, we concluded with our analysis of what we have acquired through this project and future scope.

\section{GLOBAL OVERVIEW}

We present here the context, some related work, issues, and our objectives.

\subsection{Context}

The amount of text data on the web, or stored by companies is growing continuously. In order to exploit this wealth, it is essential to extract knowledge from such data. The discipline dealing

DOI : 10.14810/ecij.2017.6401 
Electrical \& Computer Engineering: An International Journal (ECIJ) Vol.6, No.3/4, December 2017

with this type of data is called "Text Mining" includes several issues such as search indexing of documents, summary generation, creation of bots, etc. The work done during our project is part of the enrichment of the Palo IT textual and data analysis research. It aims to create a semiintelligent bot. For this project an internal R \& D was launched. This project "PetiText" (translated SmallText in English; petit=small) is based on the analysis and reasoning on short sentences to detect new facts and answer questions. It involves an analysis of data from text corpora which allows to:

- extract targeted information, sorted and added value for companies using algorithms

- search for similarities and identifying causal relationships between different facts

- detect behaviours and intentions

- $\quad$ answer questions of policy makers

- $\quad$ guide the marketing action and set up alerts to devices.

\subsection{Issues}

Faced with the growing demand of Palo IT customers to extract knowledge from their textual data, the PetiText R \& D project was launched. Indeed, these customers possess documents and tools for collecting reviews and customer complaints or employees. Hence the need to design and implement a tool for analysing this type of data. Text data poorly used by most companies, represent a wealth of information. Their analysis is a means of decision support and a strategic asset for companies. Study of Text Mining existing products shows a major flaw for processing text data written in French. This defect consists of the almost total absence of "open source" libraries incorporating the semantics of the French language. Indeed, unlike the English, we found that most libraries and tools used globally (as Clips [1], NLTK [2], etc.) to treat this type of problem are not reliable when it comes to deal in French documents. For these reasons it was decided to set up a new tool combining several text analysis methods that treats the French language, which allows the machine to reason as a little boy of two years.

\subsection{Related works}

Some authors have proposed to deal with those issues by deep learning and ontology reasoning. It was the case of [14] Patrick Hohenecker and Thomas Lukasiewicz, from Department of Computer Science University of Oxford, introduce a new model for statistical relational learning that is built upon deep recursive neural networks, and give experimental evidence that it can easily compete with, or even outperform, existing logic-based reasoners on the task of ontology reasoning. Other authors recently have proposed in [15] a model that builds an abstract knowledge graph on the entities and relations present in a document which can then be used to answer questions about the document. It is trained end-to-end: only supervision to the model is in the form of correct answers to the questions. Thuy Vu and D. Stott Parker [16] describe a technique for adding contextual distinctions to word embeddings by extending the usual embedding process - into two phases. The first phase resembles existing methods, but also constructs $K$ classifications of concepts. The second phase uses these classifications in developing refined $K$ embeddings for words, namely word $K$-embeddings. We propose to 
Electrical \& Computer Engineering: An International Journal (ECIJ) Vol.6, No.3/4, December 2017

complete these propositions with an approach to connect human language and knowledge bases (here we start with French but it must be same thing for other languages).

\subsection{Objectives}

The aim of our project was to help in all the steps of creating a semi-intelligent bot. This bot will learn facts from existing textual resources by conducting a thorough analysis. It must then be able to deduce new facts and answer open questions. To achieve this, a combination of different methods of textual data analysis was used. These methods can be grouped in three axes:

- Frequency analysis: using metrics based on the detection of global information and characteristics of a text (keywords, rare words, etc.).

- Knowledge of analysis: based Keyword Analysis and Mapping of knowledge for a classification of subjects or knowledge extraction rules (logical rules).

- Semantic Analysis: based on the analysis of the context and emotions to contextualize a given text.

\subsection{Technological choices and human resources}

The "petiText" is a project that is part of Data Science Palo IT activities leaded by three PhDs: a data science expert as supervisor Mr. Patrick LAFFITTE, then Mrs. Raja HADDAD and Mr. Yassin CHABEB. Thanks to the wealth of existing libraries in python dedicated to machine learning, the choice of that language was obvious. Regarding data storage, we used ZODB (Zope Object DataBase) which is a hierarchical and object-oriented database. It can store the data as python objects. We used Gensim [3] and Scikit-learn [4] are two python libraries that implement various machine learning methods and facilitating the statistical treatment of data. These methods of learning and statistical data computations require considerable material resources: due to the volume of data to process and especially the time computations, therefore, two remote $\mathrm{OVH}$ machines were rented. These machines have the following configurations: Machine ${ }^{\circ} 1: 8$ CPUs, $16 \mathrm{~Gb}$ of RAM and a GPU; Machine ${ }^{\circ} 2: 16$ CPUs and $128 \mathrm{~Gb}$ of RAM.

\section{TASKS CARRIED OUT}

During our project, we were able to participate in the implementation of several tasks on the textual analysis of sentences from corpus of documents to create an intelligent bot capable of answering questions in real-time interaction. The approach on which the bot was based would allow also to exploit $80 \%$ of stored data in some enterprises and generate a lot of hidden facts, this stored data is not exploited by the enterprises' businesses.

\subsection{Drawing conclusions from a set of sentences}

The objective of this set is to create and implement a formal logic model. This was achieved by combining the results of two tools. The first is used to apply a logical model to a set of sentences modelled as relationships between objects. These were extracted through the use of the second tool is the CoreNLP. Appendix A shows an example of application of our logic model on a set of sentences about the family universe/field/domain. 
Electrical \& Computer Engineering: An International Journal (ECIJ) Vol.6, No.3/4, December 2017

\subsubsection{Building a logic model}

This model is based on interpreting the world as a set of facts, and every fact is the relationship between two or more objects. Knowing that the objects of a sentence are a fact (a relationship), it is sufficient to apply logical rules that we have defined to derive and generate new information between different objects.

A simple example:

$>\quad$ Man is a creature. [Fact in input]

$>\quad$ John is a man. [Fact in input]

$\checkmark \quad$ John is a creature. [Generated fact]

To extract new information from a given set of facts, we have implemented a set of logical rules. When a rule can generate a new fact (called conclusion), or a hypothesis. Each hypothesis can become a conclusion if new facts arrive and validate it. The logical rules that we have defined in this part:

- Conclusions:

- $\quad$ If obj1 a obj2 Then obj2 is part of obj1.

- $\quad$ If obj1 is obj2 AND obj2 is obj3, Then obj1 is obj3.

- $\quad$ If obj 1 is obj2 OR obj3, Then obj1 is obj2 OR obj1 is obj3.

- If obj1 is obj2 OR obj3 AND obj2 is obj2-1 OR obj2-2 AND obj3 is obj3-1 OR obj32 ,

Then obj1 is obj2-1 OR obj2-2 OR obj3-1 OR obj3-2.

- If obj1 is obj2 OR obj3 AND obj2 is obj4 AND obj3 is obj5, Then obj1 is obj4 OR obj5.

- $\quad$ If obj1 is obj2 AND obj3 is obj2, Then obj1 AND obj3 are obj2.

- The hypotheses:

- $\quad$ If obj 1 is obj2 OR obj3 AND obj3 is obj2, Then obj3 is probably obj1.

- If obj1 is obj2 OR obj3 AND obj4 is obj5 AND obj2 Then obj4 is probably obj1.

- $\quad$ If obj 1 is obj2 OR obj3 AND obj2 is probably obj4, Then obj1 is probably obj4. 


\subsubsection{CoreNLP}

The construction of logic models from the input sentences needs first level of understanding from a syntactic and grammatical analysis. For this we used a natural language processing tool (NLP) entitled CoreNLP [5] of Stanford University. CoreNLP fetches lemmas of words, it identifies their basic form from plural or conjugation or grammatical declinations. It also explains the overall structure of the sentence analysing subject, verb, etc. It also acts as a parser adapting to the turn of phrases. Figure 1 shows an example of analysis of a sentence using CoreNLP. When edges represent the overall structure of the sentence, then the blue tags represent lemmas of different words, and those in red, give the grammatical class of a word.

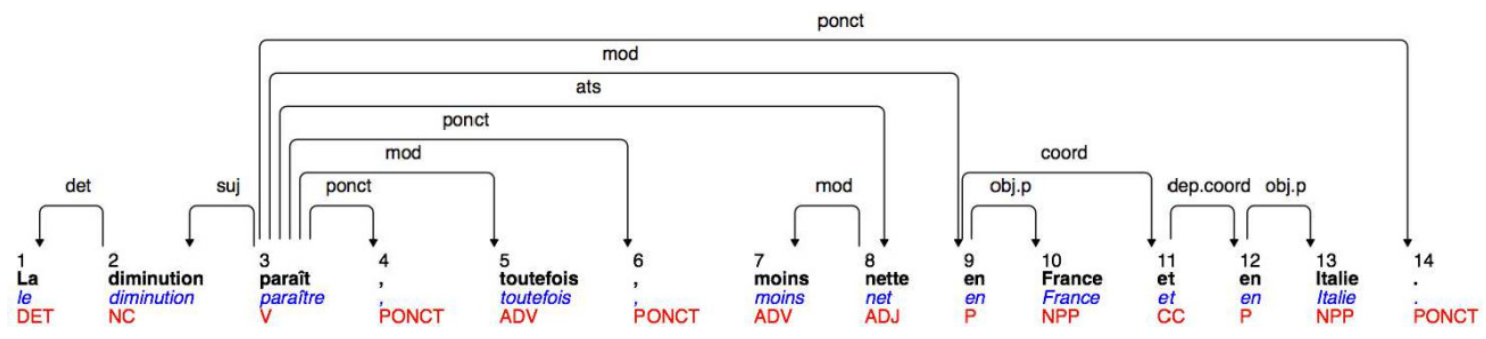

Figure 1. Example of CoreNLP analysis result

\subsection{Recover Data (The scraping [6] with XPath [7])}

Web Scraping is a set of techniques to extract the contents of a source (a website or other). The goal is to transform the data retrieved for use:

- $\quad$ For rapid integration between applications (when API is not available).

- $\quad$ Or to store this data in data base to be analysed later.

In this project we used this technique (Web Scraping) to store a maximum of French-language definitions. This is to integrate the intelligent aspect to our bot. So that it can answer questions, the meaning of words and phrases is very important, so the choice of web sites and dictionaries of synonyms has emerged. To scrabble definitions from various dictionaries of sites we used XPath. This library allows python to extract information (elements, attributes, comments, etc.) of a document through the formulation of terms including

- An axis (child or parent).

- A node (name or type).

- One or more predicates (optional).

These definitions and synonyms have served as a learning base to allow the bot to learn the meaning of words and phrases in different contexts. 
Electrical \& Computer Engineering: An International Journal (ECIJ) Vol.6, No.3/4, December 2017

\subsection{Develop statistical models and learning}

During this research project we have implemented and tested several models of text data as learning the TF-IDF, the word embeddings. The application of these two models to learn allowed us to have a clear idea about the use cases for each model.

\subsubsection{TF-IDF}

The Extracting relevant information from textual sources based on statistical models. These are used to detect rare words (therefore the most significant), to eliminate less significant as stopwords that does not depend from the context. The most commonly used technique for this is the TF-IDF [8] (Term Frequency - Inverse Document Frequency).

$$
\begin{gathered}
t f(t, d)=\frac{f_{t, d}}{\max \left\{f_{t, d}: t^{\prime} \in d\right\}} \\
\operatorname{idf}(t, D)=\log \frac{N}{|\{d \in D: t \in d\}|} \\
\operatorname{tfidf}(t, d, D)=t f(t, d) \times i d f(t, D)
\end{gathered}
$$

- $\quad \mathrm{f}$ : The frequency of the term $t$ in the document.

- $\quad$ D: All documents.

- $\quad \mathrm{N}$ : Total number of documents in the corpus $(\mathrm{N}=|\mathrm{D}|)$.

- $\quad \mathrm{d}$ : A document in all documents $\mathrm{D}$.

- $\quad \mathrm{t}$ : A term in a document.

\subsubsection{Word embeddings}

To build a learning model based on the word embeddings every sentence is converted into vector of real values. The application of a model based on the succession of several layers neural networks to detect semantics, contexts and relationships between them, and the classification of new texts through an unsupervised learning. To apply the word embeddings on a textual corpus we used different python libraries like: Gensim (Word embeddings) and Scikit-learn. They offer all necessary methods (Doc2Vec, word2Vec...).

\subsubsection{TF-IDF versus Word embeddings}

We assessed the reliability of the two learning models cited earlier. This evaluation was to be applied on a data set (20 newsgroups) 20,000 items and categories 20 (1000 items per category). For this test, TF-IDF comes at a score of $58 \%$ while the word embeddings gives a score of $98 \%$. This result supported our choice to use the word embeddings as a learning model for our bot. 


\section{INTELLIGENT BOT}

To develop an intelligent (or semi-intelligent) bot that can analyse the facts, learn and answer questions, we combined the different parts detailed in the previous section. So, our bot combines between the quality of text processing, classical and machine learning logic (semantics). Our bot mainly combines three components: logic, semantics and training/learning base. The bot must be logical, intelligent, autonomous and must render services. In our case the services are to answer questions and generate different contexts, conclusions and assumptions from the facts given to enrich its knowledge base. Aside from the natural language processing that must be done automatically with the arrival of new developments, several challenges need to be resolved to reach reliable conclusions.

\subsection{The logic}

The bot must be logical in its calculations and answers as a human, that's why a conventional logic model was developed. This model will allow to validate or not the facts that are available, based on the rules of the basic classical logic. This will argue about the facts and generate conclusions to be potentially added to the knowledge base of the bot. Before moving to the logical model, the facts, which are only phrases, must first be processed and decomposed by a natural language processing tool. Question complexity and execution time, the logic model is the simplicity of classical logic rules and is very fast because the facts are relatively simple sentences composed of a single verb, subject, complement, conjunction.

\subsection{The basic knowledge and autonomy of the bot}

The knowledge base is made up of all findings and the operative events that are valid. It is enriched when the facts arrive and conclusions are generated. The bot is then autonomous and no longer depends on human intervention as textual data sources feed the bot with new facts. Figure 2 summarizes the developments of the validation process. It must still that moment to launch the bot the first time, he already has a basic knowledge they will need to make learning so he can detect and recognize the context and thus be able to work on developments that arrive. The bot will look like a man (boy) that reasons and learns so the most logical is from a knowledge base that is based on dictionary definitions (for each word, several definitions depending on context) and synonyms. These are collected by the Scraping technique from several specialized websites. The departure of the knowledge base contains 38,565 definitions of 226,303 words.

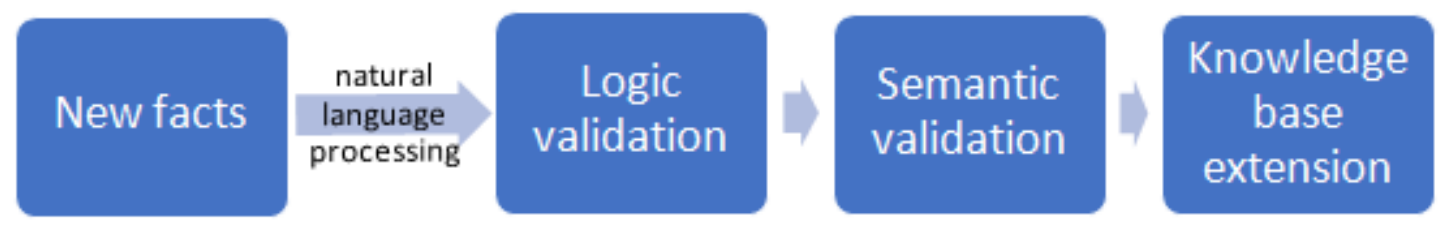

Figure 2. New facts validation process

\subsection{The intelligence}

Now that we have a logic model and an initial knowledge base, the challenge is to find a technique that will allow the bot to detect the context of new arriving facts and rank them, and this is exactly when the word embeddings is used. Recent Deep Learning idea is that the 
Electrical \& Computer Engineering: An International Journal (ECIJ) Vol.6, No.3/4, December 2017

approximate meaning of a word or sentence can be represented as a vector in a multidimensional space. The nearer vectors represent similar meanings. To do so, we used Gensim for Python designed to automatically extract topics Semantic documents, as efficiently as possible. Gensim is designed to process raw digital and unstructured text. The algorithms in Gensim, such as Latent Semantic Analysis, latent Dirichlet allocation and random projections, describe the semantic structure of documents. The latter is extracted by examining the statistical models of cooccurrences of words in a corpus of training documents. These algorithms are unsupervised, which means that no human input is required. The only input to these algorithms is the text document corpus for training the model. Gensim allows:

- Collect and process semantically similar documents.

- Analyse text documents for the semantic structure.

- Making scalable statistical semantics.

\subsubsection{Word Embeddings $[9,10]$}

Word embeddings are one of the most exciting areas of research in the field of Deep Learning. A word embeddings WE: words $\rightarrow \mathbb{R} n$ is a parameterized function that maps words in a certain language to high-dimensional vectors $(100,200$ to 500 sizes). Essentially, each word is represented by a numerical vector. For example, we might find (poire means pear in French):

- $\quad$ WE $($ "pair" $)=(0.2,-0.4,0.7, \ldots)$

- WE $($ paire" $)=(0.2,-0.1,0.7, \ldots)$

- $\quad$ WE $("$ pear" $)=(0.0-0.3 ; 0.1, \ldots)$

- $\quad$ WE $($ "poire" $)=(0.1,-0.1,0.2, \ldots)$

The purpose and usefulness of the word embeddings consist in grouping the vectors of similar words in a vector space. Mathematically, it detects similarities between different vectors. These are digital representations describing the characteristics of the word, such as context. The word embeddings has several variations including:

- the Word2vec

- the Doc2vec

\subsubsection{Word2vec [11]}

Word2vec is a two-layer neural network that processes the text. The input to the network is a text corpus and its output is a set of vectors. These include semantic features to the words in the corpus. Word2vec is not a deep neural network in itself, but it is very useful because it turns text into digital form (vectors) that the deep networks can understand. Figure 3 summarizes the process used in word $2 \mathrm{vec}$ algorithm. Words can be considered as discrete states, then simply search the transition probabilities between these states, such as, the probability that they occur 
Electrical \& Computer Engineering: An International Journal (ECIJ) Vol.6, No.3/4, December 2017

together. In this case we will have close vectors for the words in a similar context (plus the cosine is close to 1 , the more the context of these words is similar).

\section{word2vec}

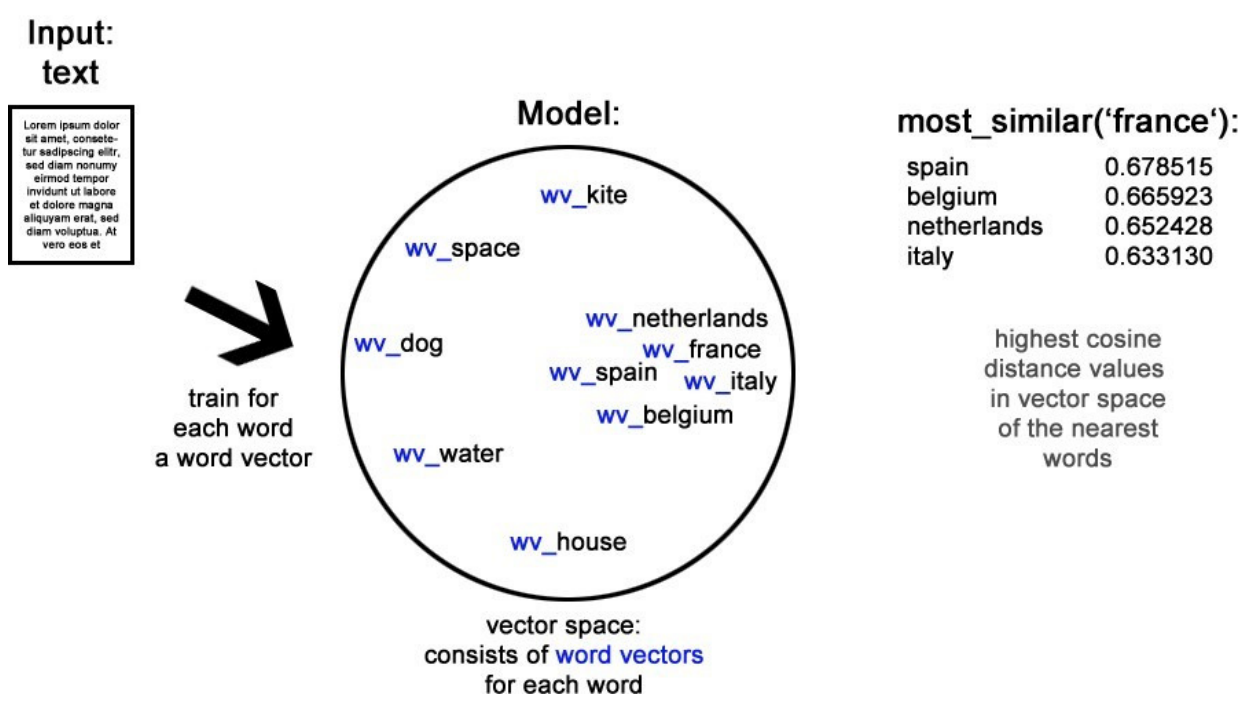

Figure 3. Description of steps word2vec (Gensim [3])

In the Mikolov [12] introduction about learning word2vec, each word is mapped to a single vector, represented by a column in a matrix. The column is indexed by the position of the word in the vocabulary. The concatenation, or the sum of the vectors is then used as a characteristic for predicting the next word in a sentence. The Figure 4 gives an example of word $2 \mathrm{vec}$ concatenation.

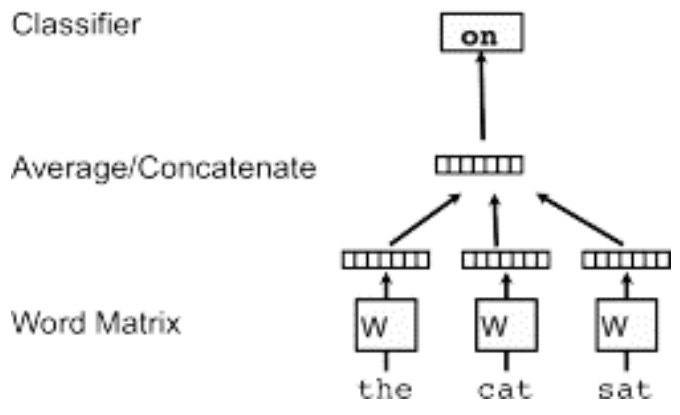

Figure 4. Word2vec example [12]

If we take into consideration enough data, use and contexts, the word2vec can make very accurate assumptions about the meaning of a word based on past appearances. These assumptions can be used to establish an association of words with other words in terms of vectors. For example: $\mathrm{W}($ 'woman') - W('man') $\approx \mathrm{W}($ 'queen') - W('king') 
Electrical \& Computer Engineering: An International Journal (ECIJ) Vol.6, No.3/4, December 2017

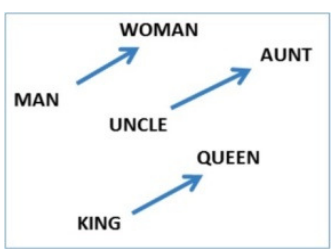

Word2vec form words by other words it detects in the input corpus. Word2vec includes two methods:

1. Continuous Bag of Words model (CBOW):

- The context (surrounding words) is used to predict the target word.

2. Skip-gram with negative sampling or skip-gram:

- A word is used to predict a target-context (surrounding words).

- This method can also work well with a small amount of training data. It can also represent words or few sentences.

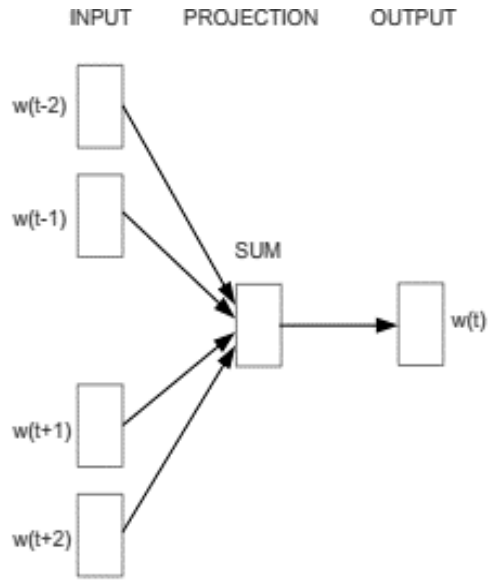

CBOW

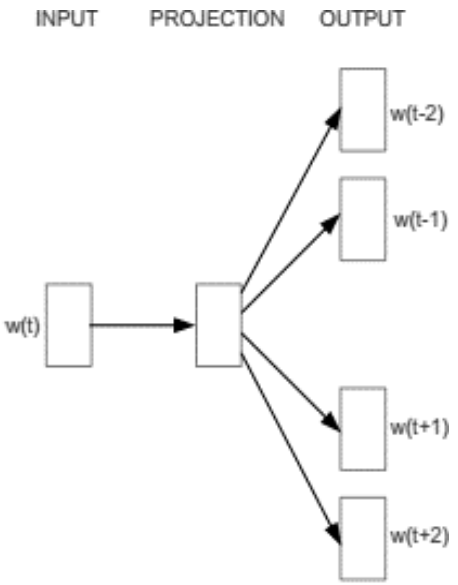

Skip-gram

Figure 5. Architecture of methods CBOW and Skip-gram. $\mathrm{w}(\mathrm{t})$ is the current word, $\mathrm{w}(\mathrm{t}-1), \mathrm{w}(\mathrm{t}-2) \ldots$ are the words surrounding the word

\subsubsection{Doc2vec [13]}

Doc2vec (Paragraph2vec) changes the algorithm word2vec (generalization of word2vec) for unsupervised learning of continuous representations for blocks of most important keywords such as sentences, paragraphs or entire documents. 


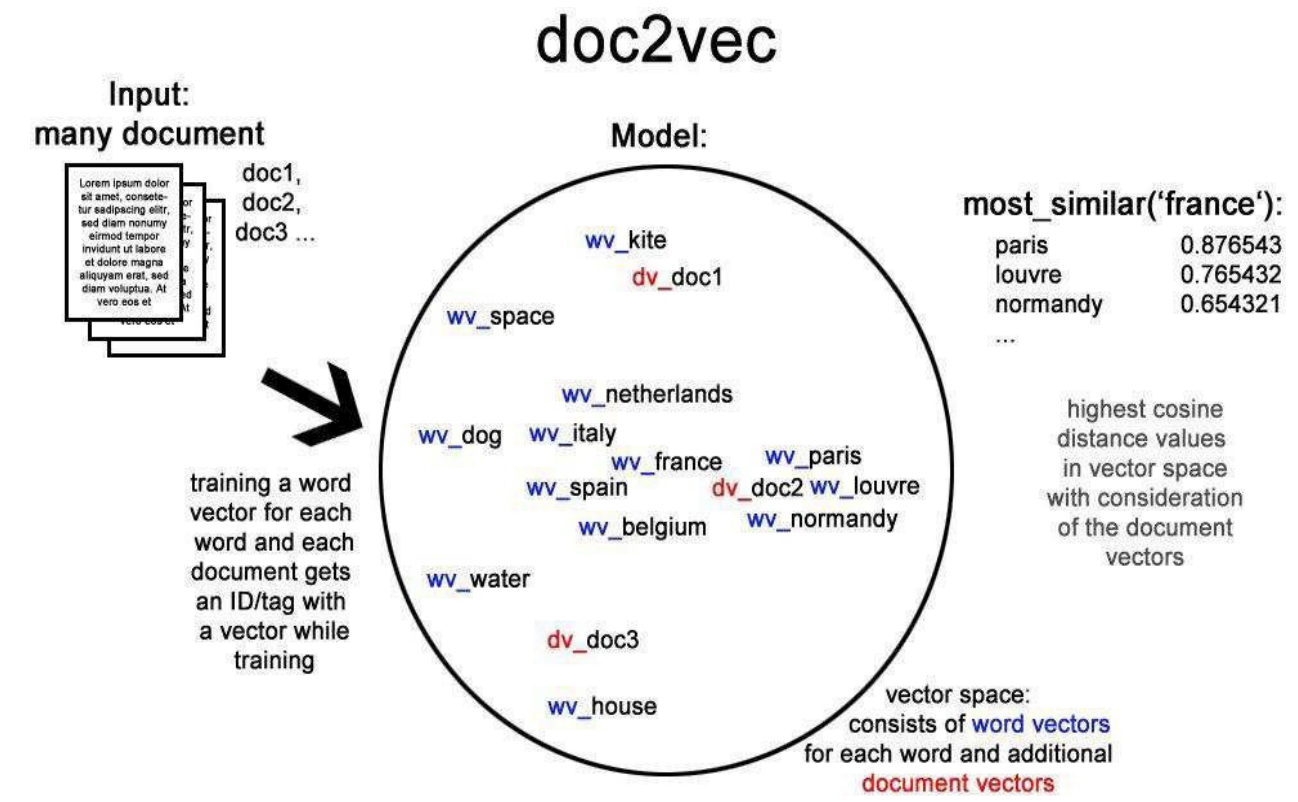

Figure 6. Description of doc2vec [3]

The Doc2vec realize a learning on a large set of documents, and creates a model of vector spaces. In this model, each document is a vector space composed by the vectors of words. Thus, to have the degrees of similarities, the method "most_similar ' uses the cosine between vectors, the higher the cosine is close to 1 , the higher the similarity is high. Figure 6 illustrates the steps of unwinding the doc2vec algorithm. To apply Doc2Vec, two methods can be used:

- distributed memory model (DM)

- distributed bag of words (DBOW)

a. Distributed memory model (DM)

It considers the vector of paragraph with the vectors of paragraph words (Word2vec) to predict the next word in a text. Using this distributed memory model (DM) comprises:

- Randomly assign a paragraph vector for each document.

- Predict the next word using the context of the word + the paragraph vector.

- Drag the window contexts on the document while the paragraph vector is fixed (therefore distributed memory)

Figure 7 illustrates the operation of distributed memory model. 
Electrical \& Computer Engineering: An International Journal (ECIJ) Vol.6, No.3/4, December 2017

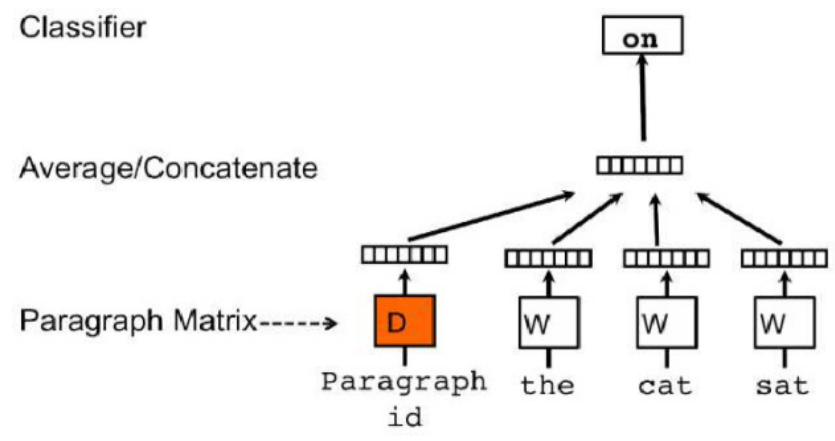

Figure 7. DM model doc2vec [12]

b. DBOW: Distributed Bag Of words

This method (DBOW) ignores the context words at the entrance. One paragraph vector predicts words in a small window. This method requires less storage and it is very similar to the method of the Skip-gram word2vec [12]. This method is less efficient than DM. However, the combination of the two methods $D M+D B O W$ is the best way to make a Doc2vec.

As shown in Figure 8, the DBOW method involves:

- Using only paragraphs vectors (No word2vec).

- Taking a window of words in a paragraph and random predict what word using paragraphs vectors (ignoring word order).

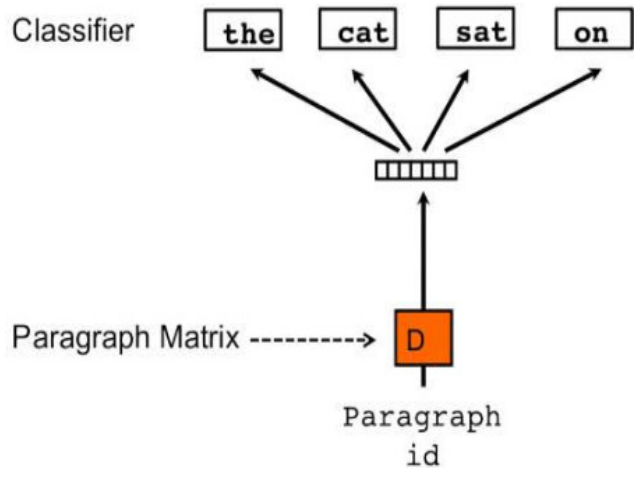

Figure 8. DBOW model doc2vec [12]

\subsection{Tests and applications}

As part of the project "PetiText" the doc2vec model is built from all available dictionaries of definitions, when each definition is tagged with a word. We proceed to the construction of the model using the method DBOW. To assign a tag to a new definition, the model infers the vector and returns the tag definitions having the highest cosine. 


\subsubsection{Learning time et scores}

With a machine having 16 CPUs and RAM $128 \mathrm{~Gb}$, the learning period of the model on 38,565 definitions of 226,303 words varies proportionally to the model parameters, the size of the generated vectors and the number of iteration of learning.

Table 1 shows the results of different tests we performed. We note that the learning time and its quality are proportional to the number of iterations of the algorithm. We also find that the best results are obtained with 200 iterations and vectors of size 200. Figure 9 illustrates the evolution of the score according to the number of iterations.

Table 1. Overview of learning times and scores of different models.

\begin{tabular}{|l|l|l|l|}
\hline Size vectors & Iterations & Learning Time & Score \\
\hline 100 & 50 & $8 \mathrm{~min}$. & $77 \%$ \\
\hline 100 & 100 & $16 \mathrm{~min}$. & $79 \%$ \\
\hline 100 & 200 & $32 \mathrm{~min}$. & $80 \%$ \\
\hline 200 & 50 & $9 \mathrm{~min}$. & $74 \%$ \\
\hline 200 & 100 & $17 \mathrm{~min}$. & $80 \%$ \\
\hline 200 & 200 & $32 \mathrm{~min}$. & $84 \%$ \\
\hline
\end{tabular}

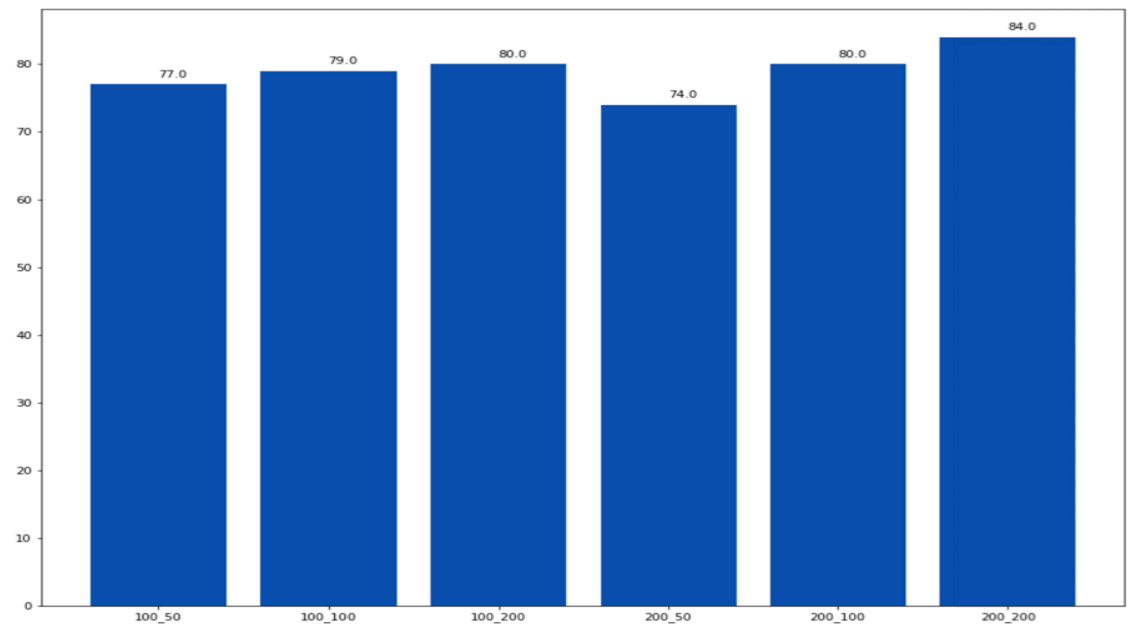

Figure 8. Scores obtained according to the size of the vectors and the number of iterations

\subsubsection{Assignment example of a tag to a new sentence}

Considering the example of a family word definition:

$$
\text { [ 'Generation', 'successive', 'down', 'ancestor', 'lined'] }
$$

The sentence has been normalized by removing stop-words, verbs are put in the infinitive and words are lemmatized.

The vector inferred by the model parameter vector size 200 corresponds to: 
Electrical \& Computer Engineering: An International Journal (ECIJ) Vol.6, No.3/4, December 2017

\section{[-2.53752857e-01 -2.71043032e-02 4.33574356e-02 -9.83970612e-02 2.55723894e-01 - 7.85913542e-02 02--5.09732738e...]}

The Figure 9 shows the tags that different models have found by calculating the cosine between the inferred vector of the previous sentence, and the vectors of all the definitions in the vector space model.

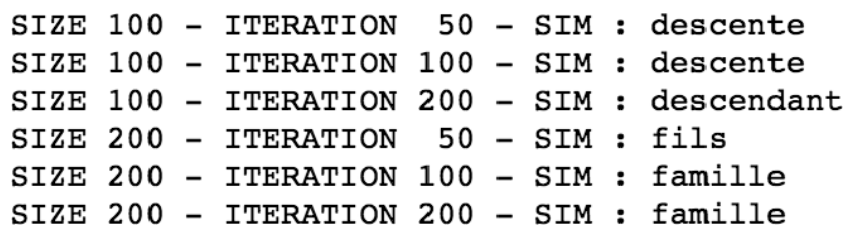

Figure 9. Search results on the context of the preceding sentence regarding the word family

For this example, we notice that the models having size vectors 200 and number of iterations 100 and 200 are the best performers, since they have been the only ones that have returned the tag "family" that corresponds to the definition of the input sentence.

Figure 10 is a screenshot of our first bot prototype. It is a French PetiText reasoner about three universes/contexts: family, abstract objects, biological organisms.

Then, we choose the family definition context then we list facts that we give to the bot.

Fact 1: a person is a man or a woman

Fact 2: a woman is female

In Figure 11, the bot starts reasoning on the facts in the order and generating conclusions and hypothesis

Generated fact 1: parents and kids are parts of the same family

Generated fact 2: a father is a male

$\ldots$

The same process was tested in real-time interaction and it works, we can also add new fact in the bottom of the screen.

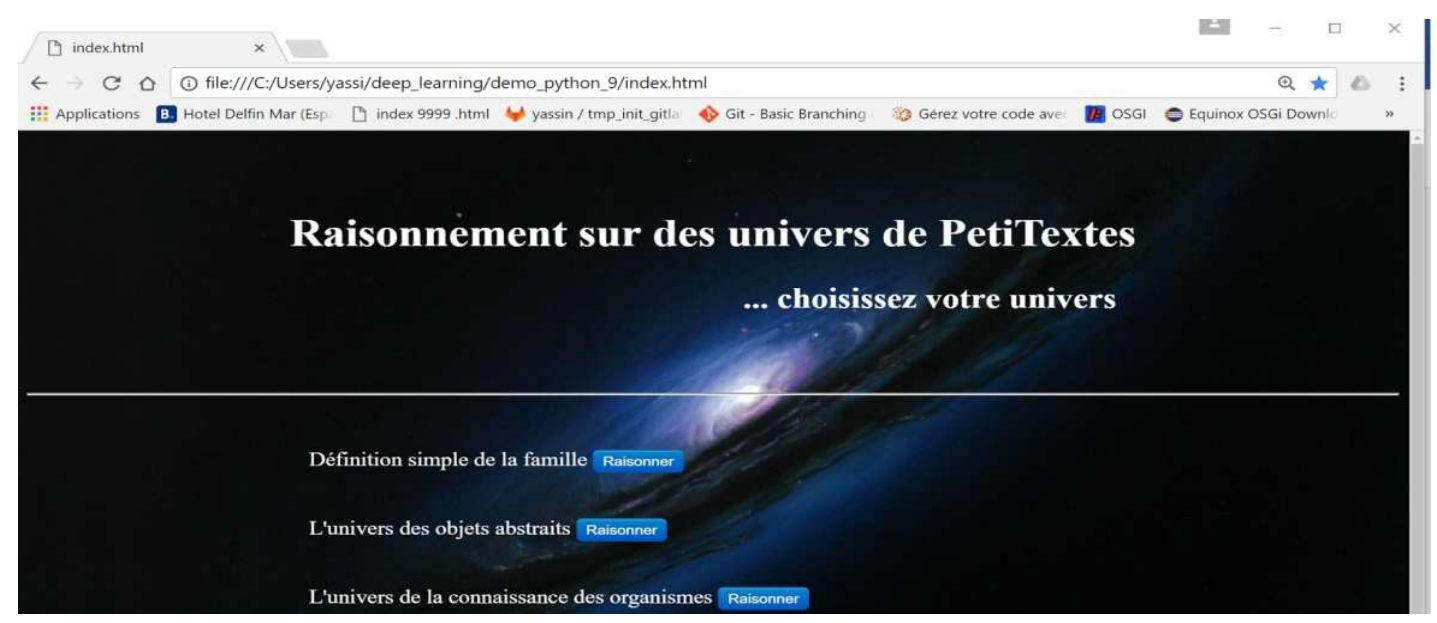




\section{Raisonnement sur la définition simple de la famille}

Une personne est un homme ou une femme.

Une femme est féminine.

Un homme est masculin.

Une famille a parents et enfants.

Un parent est un père ou une mère.

Un enfant est un fils ou une fille.

Un père est un homme.

Une mère est une femme.

Une jeune-fille est féminine.

Un garçon est masculin.

Un fils est un garçon ou un homme.

Une fille est une jeune-fille ou une femme.

Figure 9. First and second screens of bot prototype

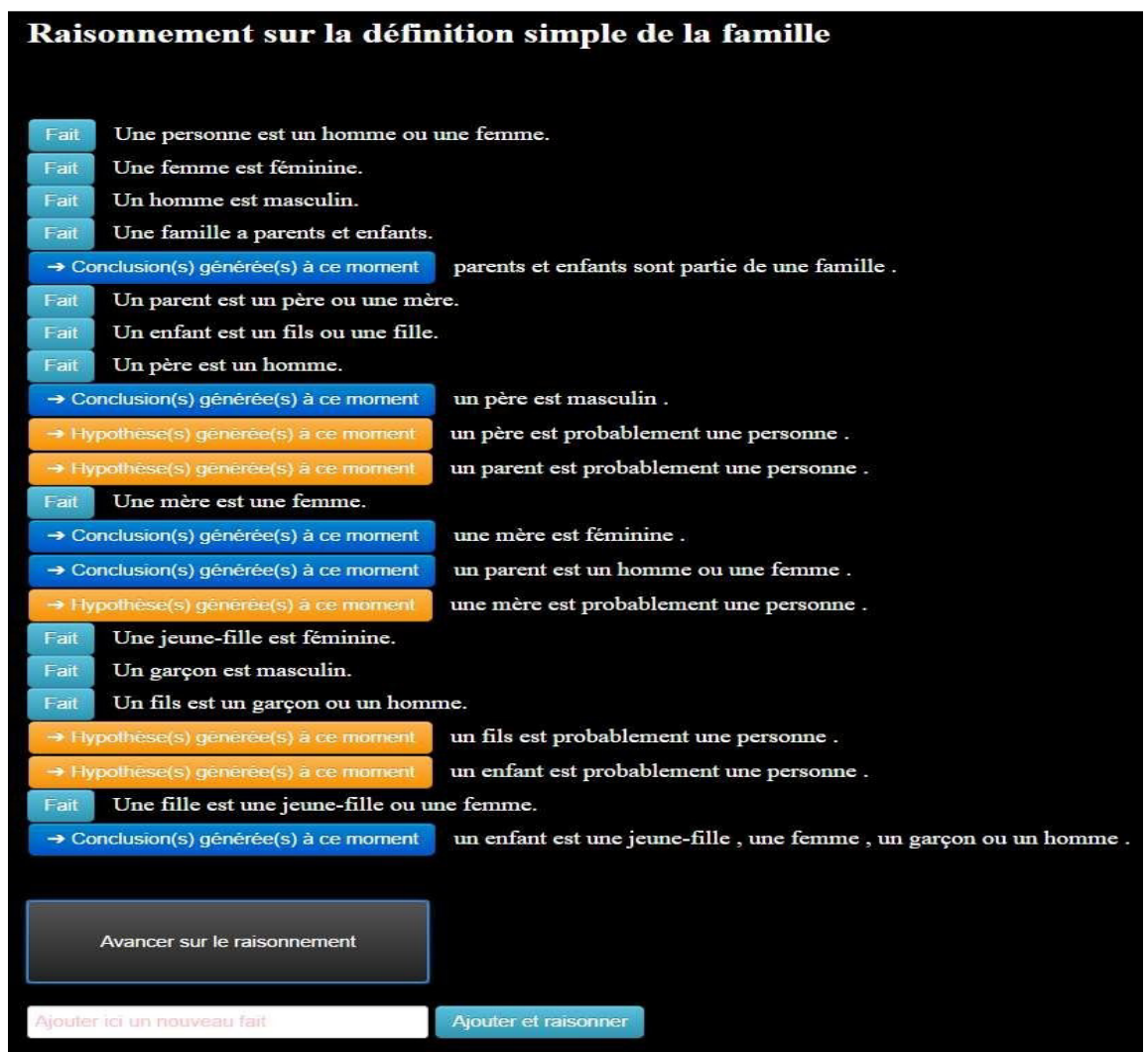

Figure 11. Reasoning screen

\section{CONCLUSIONS}

We have presented here the different steps and tools combined in order to build a semi-intelligent bot based on machine learning technologies. With a solid foundation of learning, a logic model, a 
Electrical \& Computer Engineering: An International Journal (ECIJ) Vol.6, No.3/4, December 2017

word embeddings with good scores $(\approx 84 \%)$ and improvements in current and future, we hope to combine the three parts in order to have a functional bot. We use advanced tools and technologies, they are very recent and widespread on the Data Science: the python programming language, jupyter notebook for a complete development environment, Gensim for word embeddings, and advanced tools for natural languages processing such as Clips and CoreNLP Stanford. During the project, we have used namely the methods of classification and clustering, Data Mining and Text Mining, Sentiment Analysis, and evaluations of the quality of classifiers (Reminder, Precision, F-Measure). Then come the current systems, languages and paradigms for Big Data and Advanced Big Data Analytics, that showed us especially the world of Big Data, many use cases and market opportunities. We have tested some big data architectures, including Hadoop and Spark with Python languages, Java and Scala.

As the subject of the project is part of the R\&D activities, the ultimate goal was clear and understandable, but in practice, problems on understanding how to achieve the objectives arise. Indeed, understanding how to get the value and signification of a text is not easy. Then follows the difficulty of understanding the functioning of the used methods, which algorithms to use and when to use it. Development work and tests, reading papers and publications of other researchers, and the documentation, all that was done in order to understand the subject, to progress and have good results $(\approx 84 \%)$, it was not the case at the beginning $(\approx 60 \%)$.

After months of data processing (scraping, natural language processing, data cleaning, data standardization...) and the development of logic and learning models (Word embeddings), the first results / satisfactory scores were obtained. Now, we plan to make improvements and use of new techniques for the coming months. We will use LSTM (Long Short-Term Memory), an architecture of recurrent neural networks (RNN) which should further improve the quality of prediction and classification. We plan also to finalise the integration of the bot within a Parrot drone that we controlled by voice thanks to a previous research project in order to complete a global interactive real-time interface between human and drones/robots [18]

\section{ACKNOWLEDGEMENTS}

We like to thank everyone that helped us during the current year.

\section{REFERENCES}

[1] CLiPS, https://www.clips.uantwerpen.be/PAGES/PATTERN-FR.

[2] NLTK, http://www.nltk.org/

[3] Gensim, https://radimrehurek.com/project/gensim/

[4] Scikit learn, http://scikit-learn.org/stable/

[5] CoreNLP, https://stanfordnlp.github.io/CoreNLP/

[6] Web scraping, https://www.webharvy.com/articles/what-is-web-scraping.html

[7] XPath, https://www.w3.org/TR/1999/REC-xpath-19991116/

[8] TF-IDF, http://www.tfidf.com/ 
Electrical \& Computer Engineering: An International Journal (ECIJ) Vol.6, No.3/4, December 2017

[9] Quoc V. Le \& Tomas Mikolov (2014) Distributed Representations of Sentences and Documents. CoRR abs/1405.4053

[10] Word-Embeddings, http://sanjaymeena.io/tech/word-embeddings/

[11] Word2vec and Doc2vec, http://gensim.narkive.com/RavqZorK/gensim-4914-graphic-representationsof-word2vec-and-doc2vec

[12] Quoc V. Le \& Tomas Mikolov (2014) Distributed Representations of Sentences and Documents. CoRR abs/1405.4053

[13] Representations of word2vec and doc2vec, http://gensim.narkive.com/RavqZorK/gensim-4914graphic-representations-of-word2vec-and-doc2vec

[14] Patrick Hohenecker, Thomas Lukasiewicz (2009) "Deep Learning for Ontology Reasoning”, CoRR abs/1705.10342

[15] Trapit Bansal, Arvind Neelakantan, Andrew McCallum, (2017) "RelNet: End-to-end Modeling of Entities \& Relations”, University of Massachusetts Amherst, CoRR abs/1706.07179.

[16] Thuy Vu \& Douglas Stott Parker, (2016) "\$K\$-Embeddings: Learning Conceptual Embeddings for Words using Context", HLT-NAACL, pp 1262-1267

[17] Palo IT, http://palo-it.com/

[18] Voice IT, https://github.com/Palo-IT/voice-IT

\section{AUTHORS}

Ali Rahmani, data engineer, Palo IT

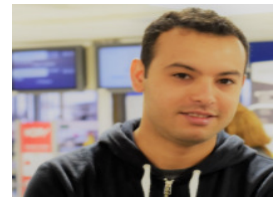

Patrick Laffitte, PhD and data science expert, Palo IT

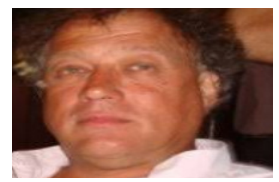

Raja Haddad, PhD and data scientist, Palo IT

Yassin Chabeb, PhD, R\&D Consultant, Palo IT
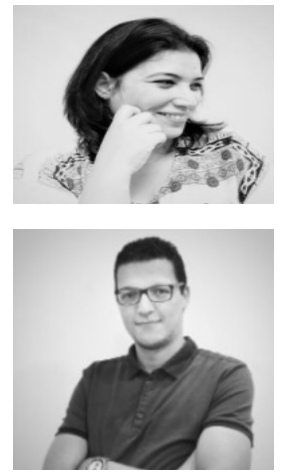